\title{
Effects of Visible Light Irradiation on Eugenol-treated Oral Mucosa
}

\author{
Seiichiro FUJISAWA, Eitoku MURAOKA, Yoshihiro NAKAZATO and Norihisa OKADA \\ Department of Oral Diagnosis, School of Dentistry, Meikai University, Keyakidai, Sakado, Saitama 350-0283, Japan \\ Corresponding author, E-mail:fujisawa@dent.meikai.ac.jp
}

Received December 22, 2004/Accepted March 25, 2005

\begin{abstract}
The purpose of this study was to evaluate the histopathological effects of eugenol (EUG) and iso-eugenol (IsoEUG) - with or without visible light (VL) irradiation - on oral mucous membranes. Oral mucous membranes of mice were applied with three agents, EUG, IsoEUG, and aceton (as the control) in the absence or presence of VL irradiation. VL irradiation resulted in more tissue damage for EUG- or IsoEUG-treated mucosa compared to corresponding compounds without VL irradiation, and that damage under IsoEUG treatment was greater than that under EUG treatment. Necrosis, but not apoptosis, was preferentially expressed in EUG- or IsoEUG-treated mucous membranes in the presence of VL irradiation.
\end{abstract}

Key words : Toxicity, Eugenols, Visible light

\section{INTRODUCTION}

EUG (4-allyl-2-methoxyphenol), an antioxidant is known to be an anti-inflammatory agent. However, when exposed to VL, it has been reported that the radical intensity and cytotoxicity of EUG aggravated inflammatory reaction ${ }^{1)}$. In addition its related compound, IsoEUG (2-methoxy-4-propenylphenol), has been found to be of higher cytotoxicity than EUG even without exposure to $\mathrm{VL}^{2)}$.

VL irradiation is widely employed in resin restoration systems in modern dentistry ${ }^{3)}$. However, there lingers a disturbing concern: the biological effects of dental materials after VL irradiation remain largely unknown ${ }^{4-9)}$. We previously reported that EUG, a component of zinc-oxide EUG cement, showed a higher cytotoxicity against human gingival fibroblast cells when VL irradiation was performed $^{10,11)}$. Also, we have demonstrated that bisEUG, which was synthesized from EUG, was far less cytotoxic at cell $^{12)}$ and tissue ${ }^{13)}$ levels due to the less prooxidative activity of bis-EUG.

This suggested that cytotoxicity was provably due to the formation of EUG semiquinone radicals, which involved the oxidation of reactive oxygen species (ROS) in living cells ${ }^{11,12,14)}$.

In this study, we investigated the cytotoxic effects of EUG and IsoEUG on the oral mucous membranes of mice in the absence or presence of VL irradiation. Cytotoxicity was evaluated by examining the morphological changes in cells and tissues, using both Hematoxylin and Eosin (H\&E) stain and TUNEL method ${ }^{15)}$.

\section{MATERIALS AND METHODS}

EUG and IsoEUG obtained from Tokyo Kasei (Tokyo, Japan) were used without further purifica- tion. The purity of EUG and IsoEUG were approximately $>97 \%$. Six adult mice (ICR, 8 weeks) were divided into two groups. Round filter papers of $1-\mathrm{cm}$ diameter (Toyo Filter Paper, Tokyo, Japan) were prepared to be immersed in $0.5 \mathrm{ml}$ of EUG or IsoEUG. For one group of mice, the filter paper with a test agent was applied to cheek mucous membranes under anesthesia (diethyl ether, Wako, Japan). For the other group of mice, they were likewise applied with a test agent but coupled with five minutes of VL irradiation. For VL lamp, Astral ${ }^{\circledR}$ (Litema, Germany, $\lambda_{\max }=400-500 \mathrm{~nm}$, irradiation dose $400 \mathrm{~mW} / \mathrm{cm}^{2}<$ ) was used. According to the animal experiment guidelines of Meikai University School of Dentistry, the mice were sacrificed and the cheeks excised. Following which, samples were immediately fixed in $10 \%$ formalin for more than two hours at room temperature. They were studied histologically by H\&E stain, and morphological changes were also investigated using TUNEL method with ApopTag ${ }^{\circledR}$ kit $^{16-19)}$ (Intergen Company, New York).

\section{RESULTS}

It was found that VL irradiation had adverse effects on EUG- or IsoEUG-treated oral mucosa of mice, when compared with corresponding ones without VL irradiation. In IsoEUG-treated buccal epithelium, a relatively severe tissue damage was recognized with the naked eye. As for EUG-treated buccal epithelium, cellular edema was induced by VL irradiation, but increase in intracytoplasmic granules indicated that only a slight inflammation occurred (Figs. 1A and 2A). After EUG-treated mucosa of mice was VL-irradiated for five minutes, the moderate cellular edema extended widely and the appearance of hazy cells boundary suggested a strong inflammatory 


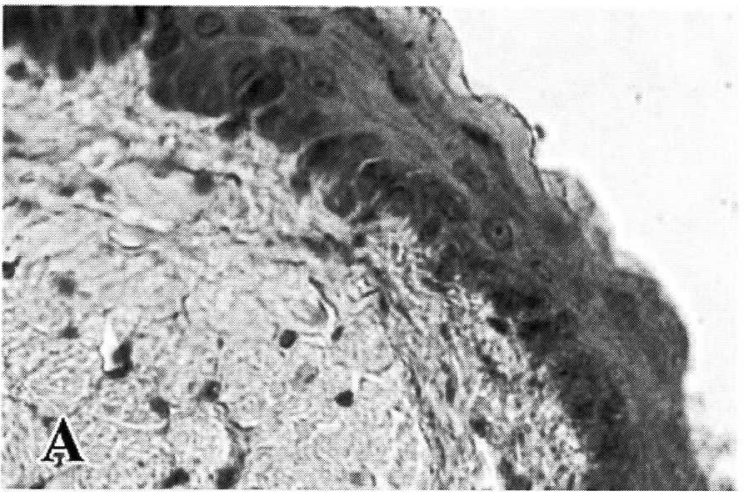

Cont

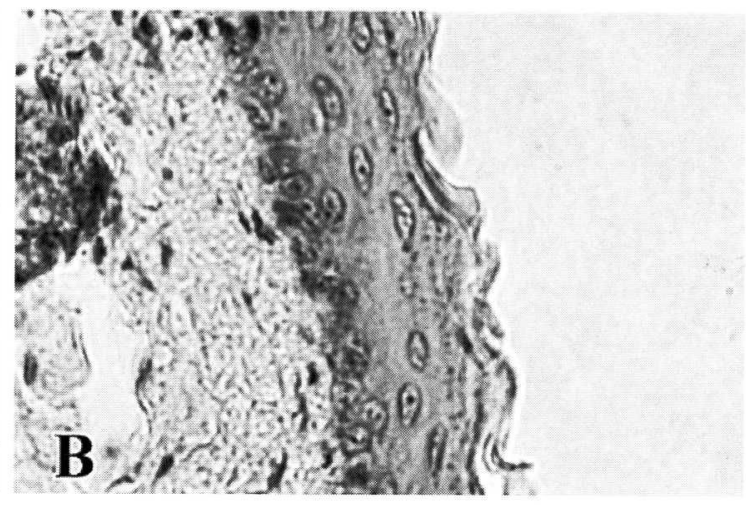

VL Cont

Fig. 1 (A) Normal histological architecture of oral mucosa of mouse. (B) Buccal epithelium following VL irradiation period of 5 minutes. Similar appearance to that of normal oral mucosa of mouse is shown in (B). H\&E stain. Original magnification $\times 500$.

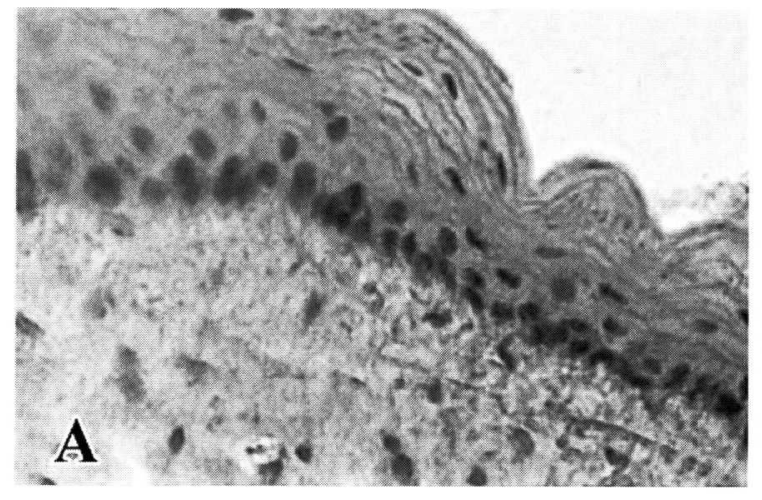

EUG

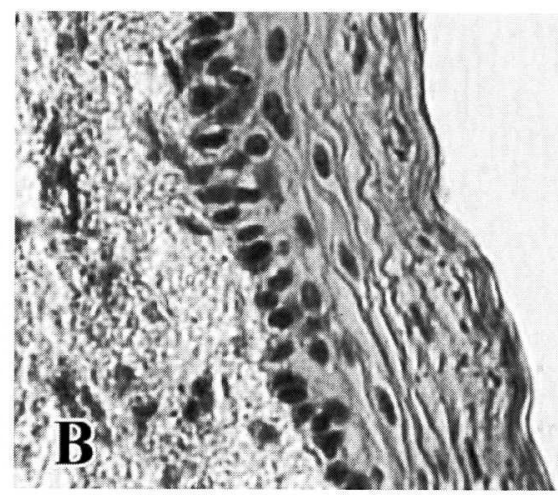

VL EUG

Fig. 2 Buccal epithelium following EUG application. (A) The light cellular edema and keratohyalin granules increased apparently. (B) Additionally, VL irradiation period of 5 minutes led to formation of moderate cellular edema and resulted in reduced staining capacity. H\&E stain. Original magnification $\times 500$.

response (Figs. $1 \mathrm{~B}$ and $2 \mathrm{~B}$ ). Under similar conditions, IsoEUG-treated samples presented a marked thickening of epithelium and the junction between cells appeared to be diffusely separated by an edema fluid, undergoing dissolution and adopting a latticelike appearance - hence suggesting an acute inflammation in this region (Figs. 1A and 3A). Furthermore, IsoEUG treatment with VL irradiation resulted in severe cellular edema together with nucleus or cell dissolution. However, there was, in part, the appearance of dispersed flattened prickle cells and a prominent granular layer in this region - hence suggesting a recovering stage (Figs. $1 \mathrm{~B}$ and $3 \mathrm{~B}$ ). In all of the samples tested, the basal cell layer showed histological integrity.

TUNEL method was performed using ApopTag ${ }^{\circledR}$ kit. In IsoEUG-treated region, the average cell size was bigger than that treated by EUG - indicating a strong inflammatory response to IsoEUG. Iso-EUG with VL irradiation showed a typical necrosis of the epithelium (Figs. 5 and 6). In each sample, the uppermost layer of epithelium stained by TUNEL method indicated a biological event similar to that characterized using H\&E stain. In addition, EUG- or IsoEUG-treated regions appeared to present a compaction of chromatin-like substances. Also, following VL irradiation, these EUG- or IsoEUG-treated regions showed an increase in staining capacity near the keratinized layer. However, the compaction of chromatin-like substances was not found in samples treated only by VL irradiation (Figs. 4, 5, and 6). 


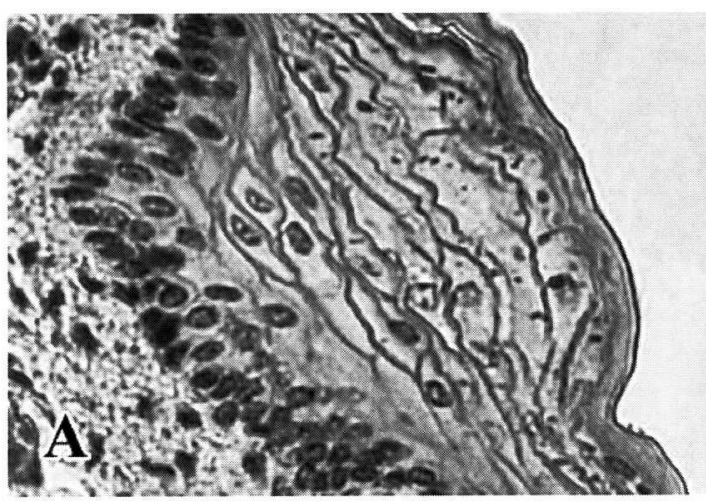

IsoEUG

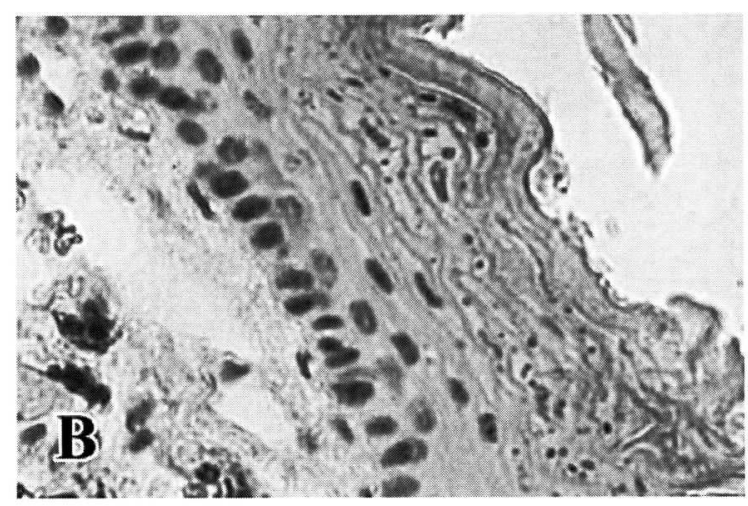

VL IsoEUG

Fig. 3 Buccal epithelium following IsoEUG application. (A) Interrupted intercellular bridge and disintegrated nuclei of prickle cells indicated a severe cellular edema. (B) Additionally, VL irradiation period of 5 minutes dispersed flattened prickle cells in deep area, indicating a severe inflammation. H\&E stain. Original magnification $\times 500$.

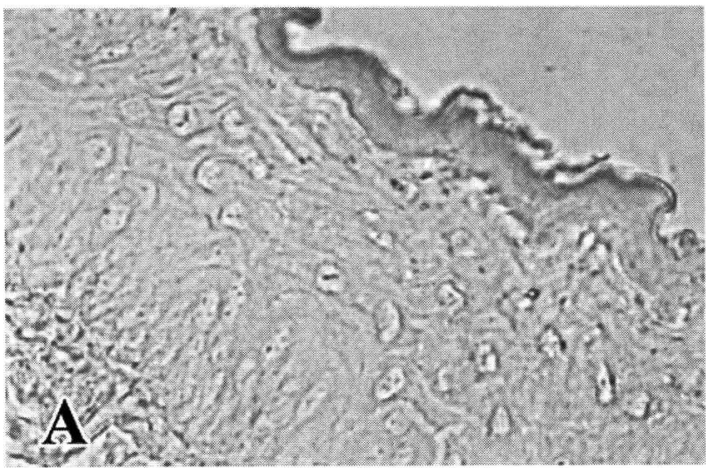

Cont

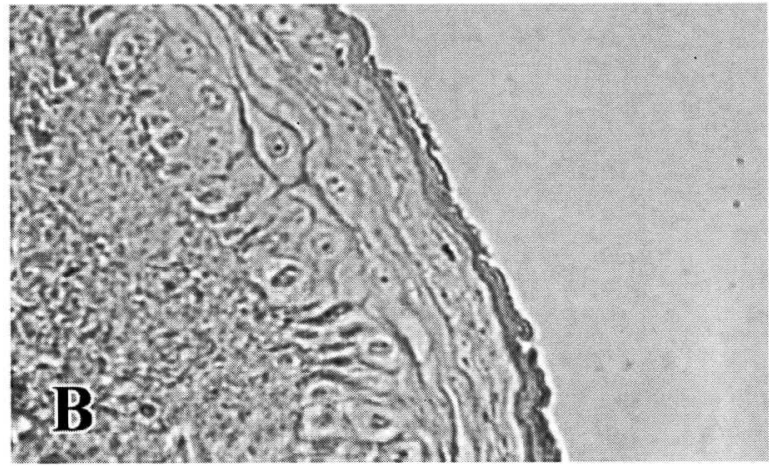

VL Cont

Fig. 4 (A) Normal histocytochemical appearance of oral mucosa of mouse. (B) Buccal epithelium following VL irradiation period of 5 minutes. Similar appearance to that of normal of oral mucosa is shown in (B). TUNEL stain. Original magnification $\times 500$.

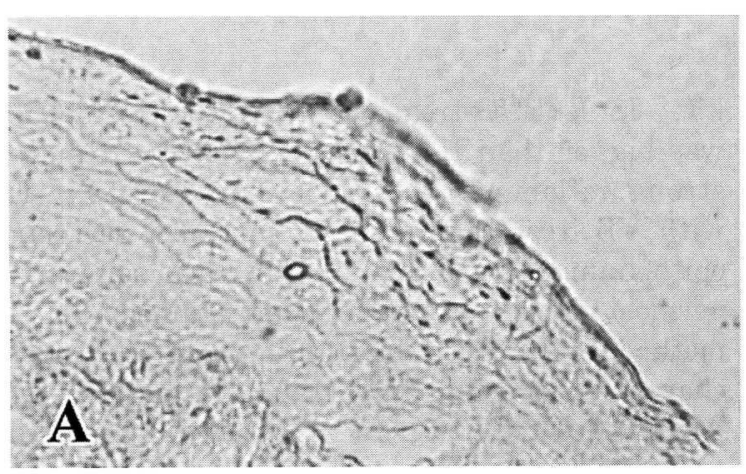

EUG

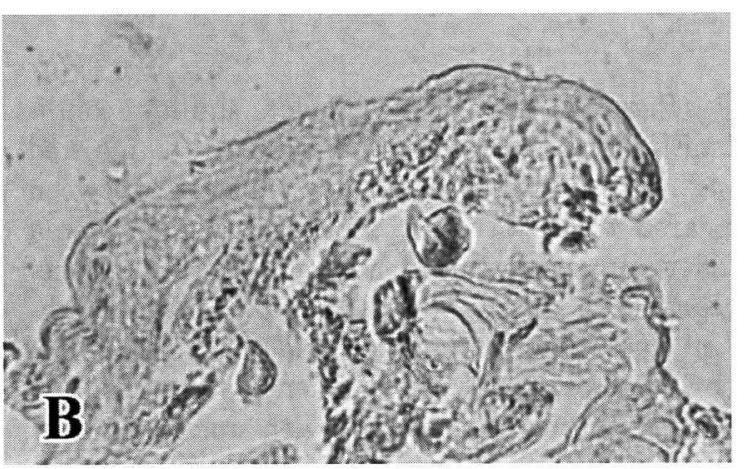

VL EUG

Fig. 5 Buccal epithelium following EUG application. (A) Interrupted intercellular bridge and compact chromatin near keratinized layer are clearly shown. (B) Additionally, VL irradiation period of 5 minutes resulted in thickening of epithelium and disappearance of cells. TUNEL stain. Original magnification $\times 500$. 


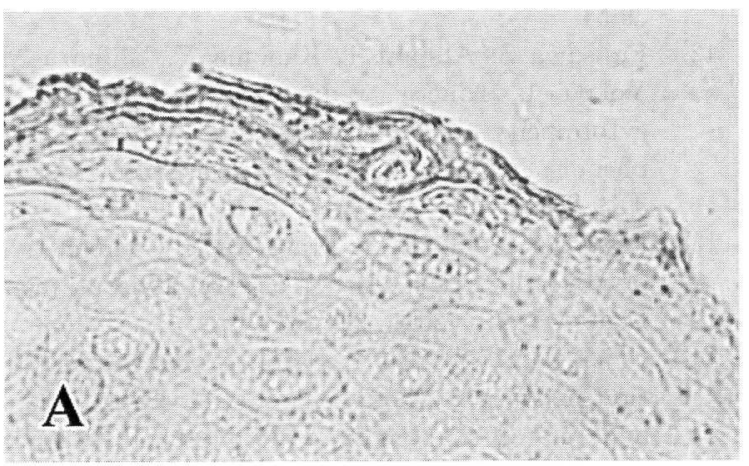

IsoEUG

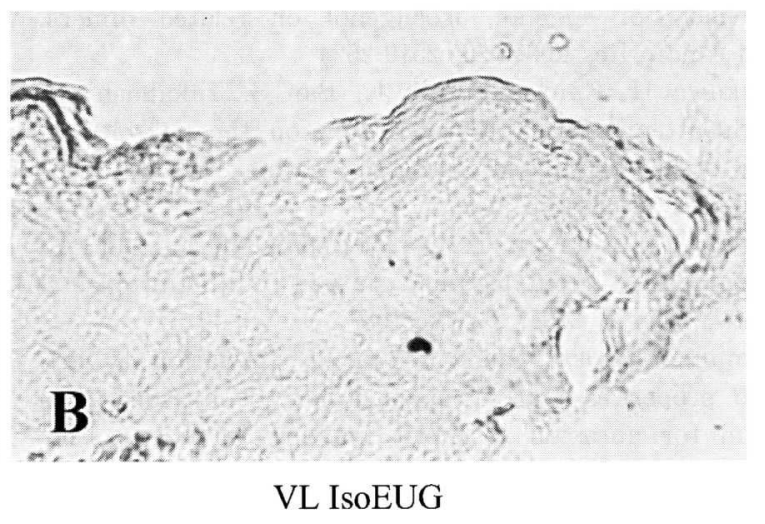

Fig. 6 Buccal epithelium following IsoEUG application. (A) Increase in staining capacity near the keratinized layer and swelling of prickle cells are clearly shown. (B) Additionally, VL irradiation period of 5 minutes resulted in marked thickening of epithelium without any cell existence. TUNEL stain. Original magnification $\times 500$.

\section{DISCUSSION}

VL is widely and frequently used as the curing system for resin restorations in modern dentistry. Though usually known to be harmless, reports have shown that VL aggravated the inflammatory reaction of EUG-applied culture cells ${ }^{1,11)}$. And at cell level its isomer, IsoEUG, indicated a cytotoxicity level very much higher than that of $\mathrm{EUG}^{2)}$. Against this background, we designed our investigation on cytotoxicity at tissue level, but not at cell level.

VL-irradiated region treated by EUG or IsoEUG showed a stronger inflammatory response than that with reagents alone. In VL-irradiated IsoEUG samples, the histological appearance of a partial recovery stage was observed. This meant that basal layer cells probably migrated to the prickle layer of flattened cells - which therefore could remain intact even when VL irradiation was performed. Strictly speaking, we must consider the duration of reaction time, turnover rate of oral mucosa of mice, and quantity or quality of VL. Although the strength of inflammation could be correlated to the reaction time of VL irradiation, the exposure was but a few minutes - hence the turnover rate of oral epithelium was most likely not affected.

In general, apoptotic cells are scarce in oral epithelium, but they make programmed cell death regularly ${ }^{16,17)}$. We performed TUNEL method using the ApopTag $^{\circledR i}$ kit, through which a proliferative zone was found in the basal layer of epithelium. This meant that cells altered to a flattened shape, disintegrated, then migrated toward the surface to become terminally differentiated when keratinization took place ${ }^{13)}$. The TUNEL method stained evidently only the nuclei at the uppermost layer of epithelium ${ }^{20)}$. Similar findings were also observed in the control group. However, when EUG was applied to an area probably under superficial layers and about 3-5 cells' thickness, the biological event obtained could be a keratinization of epithelium. It should be noted that the size, shape, and contour of cells using TUNEL method were examined carefully, and were also compared with corresponding findings in H\&E-stained cells. The findings obtained from TUNEL method did not differ from those obtained by H\&E stain.

Production of ROS and formation of intermediates from EUG or IsoEUG have been reported to be linked to cytotoxicty ${ }^{1,11,13)}$. Thus, severe tissue damage in VL-irradiated EUG- or IsoEUG-treated samples in the present study might be associated with ROS and/or the formation of their intermediates.

\section{CONCLUSION}

Tissue damage in IsoEUG samples was markedly greater than in EUG samples even without VL irradiation, and the potency of toxicity for both compounds was further enhanced by VL irradiation. Histopathological examinations using both $\mathrm{H} \& \mathrm{E}$ stain and TUNEL method suggested that the biological event characterized was predominantly a necrosis, not an apoptosis.

\section{ACKNOWLEDGEMENTS}

This study was supported in part by a Grant-in-aid from the Ministry of Education, Science, Sports and Culture of Japan (S. Fujisawa, No. 14571859).

\section{REFERENCES}

1) Atsumi T, Iwakura I, Fujisawa S, Ueha T. Reactive oxygen species generation and photo-cytotoxicity of eugenol in solutions of various $\mathrm{pH}$. Biomaterials 2001; 22: $1459-1466$.

2) Atsumi T, Fujisawa S, Satoh K, Sakagami H, Iwakura I, Ueha T, Sugita Y, Yokoe I. Cytotoxicity and radical 
intensity of eugenol, isoeugenol or related dimers. Anticancer Res 2000; 20: 2519-2524.

3) Arikawa H, Kanie T, Fujii K, Ban S, Takahashi H. Light-attenuating effect of dentin on the polymerization of light-activated restorative resins. Dent Mater J 2004; 23: 467-473.

4) Fujisawa S, Atsumi T. Cytotoxicities of a 4-META/ MMA-TBBO resin against human pulp fibroblasts. Dent Mater J 2004; 23: 106-108.

5) Tamura Y, Yokoyama A, Watari F, Kawasaki T. Surface properties and biocompatibility of nitrided titanium for abrasion resistant implant materials. Dent Mater J 2002; 21: 355-372.

6) Watanabe $\mathrm{T}$, Ban $\mathrm{S}$, Ito $\mathrm{T}$, Tsuruta $\mathrm{S}$, Kawai $\mathrm{T}$, Nakamura H. Biocompatibility of composite membrane consisting of oriented needle-like apatite and biodegradable copolymer with soft and hard tissues in rats. Dent Mater J 2004; 23: 609-612.

7) Tomiyama K, Mukai Y, Okada S, Negishi H, Fujihara T, Kawase T, Ueda M, Nakagawa S, Teranaka T. Durability of FTLA treatment as a medicament for dentin hypersensitivity: Abrasion resistance and profiles of fluoride release. Dent Mater J 2004; 23: 585-592.

8) Sugawara A, Fujikawa K, Takagi S, Chow LC, Nishiyama M, Murai S. Histopathological and cell enzyme studies of calcium phosphate cements. Dent Mater J 2004; 23: 613-620.

9) Hashimoto $Y$, Nakamura M. Cytocompatibility and viscoelastic properties of phthalate ester-free tissue conditioners. Dent Mater J 2004; 23: 412-418.

10) Fujisawa S, Kashiwagi $Y$, Atsumi $T$, Iwakura I, Ueha T, Hibino Y, Yokoe I. Application of bis-eugenol to a zinc oxide eugenol cement. J Dent 1999; 27: 291-295.

11) Fujisawa S, Atsumi T, Kadoma $Y$, Sakagami H. Antioxidant and prooxidant actions of eugenol-related compounds and their cytotoxicity. Toxicology 2002; 177:
39-54.

12) Fujisawa S, Atsumi $T$, Kadoma $Y$, Ishihara $M$, Ito $S$, Yokoe I. Kinetic radical scavenging activity and cytotoxicity of 2-methoxy- and 2-t-butyl-substituted phenols and their dimers. Anticancer Res 2004; 24: 3019-3026.

13) Fujisawa S, Okada N, Muraoka E. Comparative effects of eugenol to bis-eugenol on oral mucous membranes. Dent Mater J 2001; 20: 237-242.

14) Fujisawa S, Atsumi T, Satoh K, Sakagami H. Interaction between 2-ethoxybenzoic acid (EBA) and eugenol, and related changes in cytotoxicity. J Dent Res 2003; 82: $43-47$.

15) Kashiwagi Y. A cytotoxic study of eugenol and its ortho dimer (bis-eugenol). Meikai Daigaku Shigaku Zasshi 2000; 29: 176-188.

16) Majno G, Joris I. Apoptosis, oncosis, and necrosis: An overview of cell death. Am J Pathol 1995; 146: 3-15.

17) Labat-Moleur F, Guillermet C, Lorimier P, Robert C, Lantuejoul S, Brambilla E, Negoescu A. Tunel apoptotic cell detection in tissue sections: Critical evaluation and improvement. J Histochem Cytochem 1998; 46: 327-334.

18) Thiry M. Highly sensitive immunodetection of DNA on sections with exogenous terminal deoxynucleotidyl transferase and non-isotopic nucleotide analogues. J Histochem Cytochem 1992; 40: 411-419.

19) Ansari B, Coates PJ, Greenstein BD, Hall PA. In situ end-labeling detects DNA strand breaks in apoptosis and other physiological and pathological states. J Pathol 1993; 170: 1-8.

20) Gavrieli Y, Sherman Y, Ben-Sasson SA. Identification of programmed cell death in situ via specific labeling of nuclear DNA fragmentation. J Cell Biol 1992; 119: 493-501. 